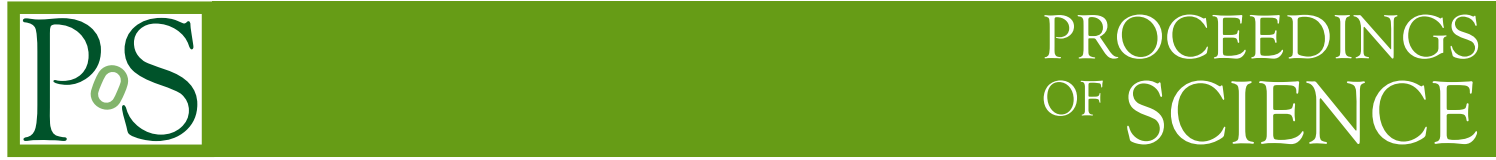

\title{
Air Shower Simulations for INCA II experiment, by using CORSIKA
}

\section{Wara Chamani}

Carrera de Física, Universidad Mayor de San Andrés, La Paz, Bolivia

E-mail: nosferatum_777@yahoo.es

In order to evaluate the sensitivity of INCA II experiment to detect Gamma Ray Burst (GRB) with energies $E>1 \mathrm{GeV}$, simulations of electromagnetic showers in the atmosphere has been performed by using the CORSIKA code. The INCA II was renewed since september, 2009, it uses some detectors of BASJE EAS array operating at Mt. Chacaltaya employing the single particle technique. 


\section{Introduction}

Gamma-ray bursts (GRBs) are the most energetic and brightest events in the universe. They are mainly observed by satellite-on-board detectors, and the most energetic components can be detected at high mountains. The INCA experiment (a collaboration between Bolivia, Italy and Japan) started from 1996 with 12 scintillator modules of $2 \times 2 \mathrm{~m}^{2}$ area, distributed over a $20 \times 20$

$\mathrm{m}^{2}$ area. From 2009, we renewed the experiment by using 49 particle detectors of BASJE(Bolivian Air Shower Joint Experiment) at Mount Chacaltaya (5200 m a.s.1.) distributed over a 700 x $500 \mathrm{~m}^{2}$ area(fig.1), namely INCA II. The aim of INCA II is to observe the high energy components of GRBs coincident with satellite and other experiments, by detecting particles with energies above $1 \mathrm{GeV}$ using the single particle technique. In order to evaluate the sensitivity of INCA II, in this work we show simulations results of electromagnetic showers in the atmosphere produced by photons in the $1 \mathrm{GeV}-1 \mathrm{TeV}$ energy range, using the CORSIKA code.

\section{Simulation and Sensitivity}

\subsection{Photon Shower Simulations}

High energy GRBs can be observed with an air shower array at high altitude, by detecting secondary particles of electromagnetic showers developed in the atmosphere. We carried out a simulation study in order to evaluate detection sensitivities of INCA II using CORSIKA. For this purpose, is chosen basically a combination between low energy hadronic interaction model and high energy hadronic interaction model as GHEISHA and QGSJETII in CORSIKA, because the first one can treat energies below $80 \mathrm{GeV}$ and the second one above $10^{11} \mathrm{eV}$, also the EGS4 and NKG options for pure electromagnetic showers were enabled for a full Monte Carlo simulation.

\subsubsection{Particle Distributions}

The simulations were performed for a primary photon with vertical incidence of zenith angle $\theta=0^{\circ}$ with a spectral index $\alpha=2$ that it takes into account the absorption in the intergalatic space and the energy cut-off value for detectors is $0.01 \mathrm{GeV}$. In order to compare the sensitivity at high altitude the figure 2 depicts charged particle distributions as a function of the photon energy at $5200 \mathrm{~m}$ a.s.l in Chacaltaya and $3500 \mathrm{~m}$ a.s.l in La Paz, also is showed the distribution of particles produced on the shower at level of Chacaltaya.

The longitudinal development of secondaries particles of different primary photon energy is showed in the figure 3, there are distributions for gammas, electrons, positrons and mean charged particles. The figure 4 shows the longitudinal distribution of charged particles given by Gaisser-Hillas function and the NKG lateral distribution of electrons all of them at level of Chacaltaya.

\subsection{Evaluation of sensitivity}

The evaluation of sensitivity of INCA II to detect a gamma ray burst depends of different burst parameters. Considering the differencial energy spectrum for a GRB is given by:

$$
\frac{d N}{d E}=k E^{-\alpha}\left[\frac{\text { photons }}{c m^{2} s G e V}\right]
$$




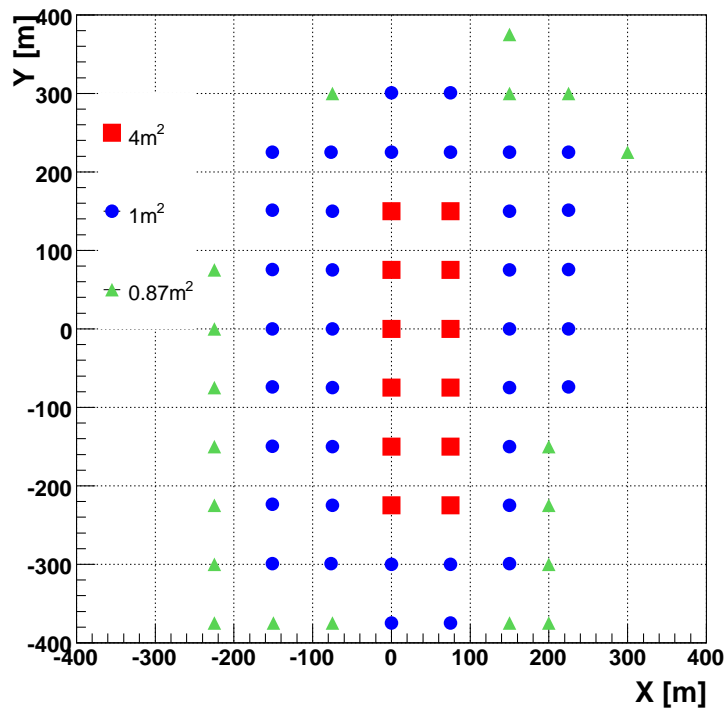

Figure 1: BASJE air shower array. The red squares and blue points represent the scintillator modules that INCA II employs.

extending from $1 \mathrm{GeV}$ up to a cutoff energy $E_{\max }$ with a time duration $\Delta t$. The signal events $N_{s}$ due to the burst is given by:

$$
N_{s}=k \Delta t \int_{1 G e V}^{E_{\max }} A_{e f f}(E) E^{-\alpha} d E
$$

where $A_{e f f}$ is the effective area of detector.

\subsubsection{Effective Area}

According to the statistical analysis given in reference 3 on effective area of detector, we consider a relation between effective area and mean number charged particles $\left(\bar{N}_{e}\right)$ produced by a photon of energy $E$ reaching the level of observation is given by:

$$
A_{e f f}(E, \theta) \approx A_{d} \cdot \bar{N}_{e}(E, \theta) \cdot \cos \theta
$$

where $A_{d}$ is the sensitive area of the detector. Since INCA II works in single coincidence mode with 49 scintillator modules( 12 of $4 m^{2}$ and 37 of $1 \mathrm{~m}^{2}$ area), we can evaluate the total effective area as:

$$
A_{e f f}=85 \cdot \bar{N}_{e}(E, \theta) \cdot \cos \theta
$$

The figure 5 shows the effective area of INCA II compared to INCA to detect primary gamma rays using the single particle mode for vertical incidence and zenith angle of $30^{\circ}$. The effective area of INCA II is 1.8 times more sensitive than INCA. 

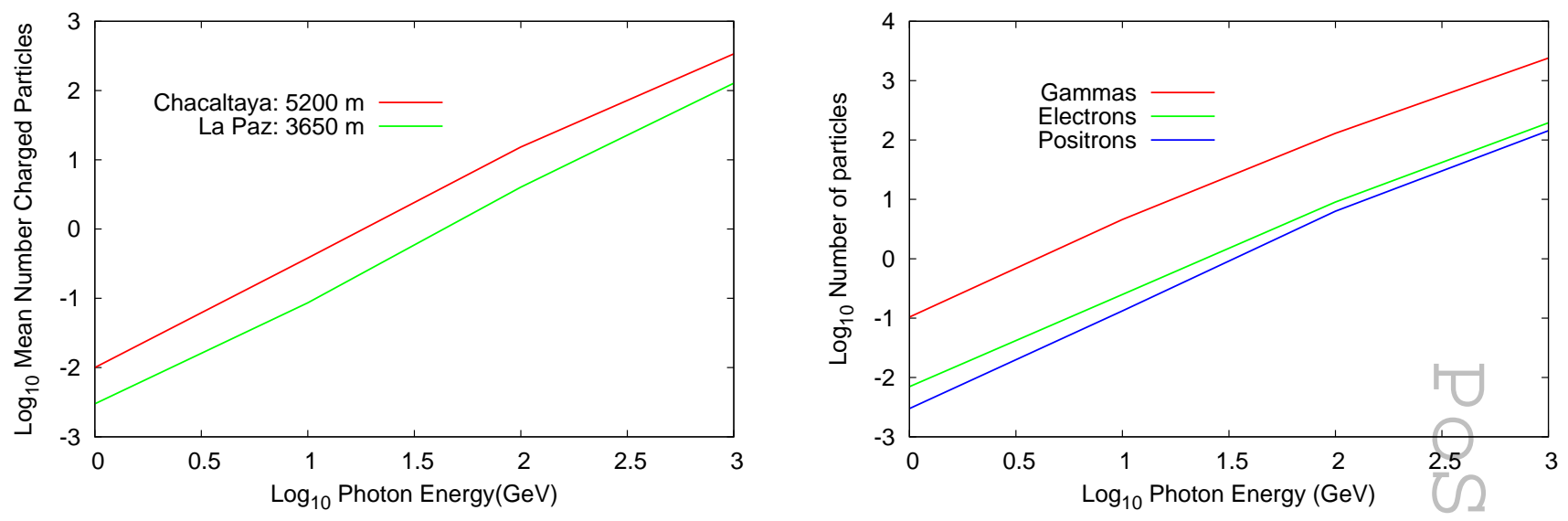

Figure 2: Left: The mean number of charged particles reaching the levels of $5200 \mathrm{~m}$ and $3500 \mathrm{~m}$, generated by a primary photon with vertical incidence in the atmosphere, as a function of the photon energy. Right: Secondary particles generated at level of Chacaltaya.

\subsubsection{GRB signal}

Working in single particle mode, a GRB signal is detectable with a statistical significance $n=4$ standard desviations. The number of detected particles $N_{s}$ has to be significantly larger than the background statistical fluctuations $\sigma_{b}=\sqrt{A_{d} B \Delta t}$, where $\mathrm{B}$ is background rate and $\Delta t$ is the GRB duration, then we have $N_{s} / \sigma_{b}>n$.

For the following calculations, is necesary to mention that the mean measured background single particle counting rate at $5200 \mathrm{~m}$ a.s. 1 is $B=500$ counts $\mathrm{m}^{-2} \mathrm{~s}^{-1}$, and for determining the minimum value of the coefficient spectrum $k$ for a detectable burst, we have to set $n=4$ and we get the next relation:

$$
k \Delta t \int_{1 G e V}^{E_{\max }} A_{d} \bar{N}_{e}(E, \theta) \cos \theta E^{-\alpha} d E=4 \sqrt{A_{d} B \Delta t}
$$

\subsubsection{Energy Fluence}

In order to evaluate the minimun energy fluence that INCA II can observe, we assume a burst giving a gamma-ray flux at the top of the atmosphere as (2.1) in the energy range $1 \mathrm{GeV} \div E_{\max }$,

$$
F=k \Delta t \int_{1 G e V}^{E_{\max }} E^{-\alpha+1} d E
$$

The figure 6 shows the minimun energy fluences for a detectable gamma-ray burst of time duration $\Delta t=1 \mathrm{~s}$ as a function of $E_{\max }$, for two zenith angles. With the new array of INCA II the minimun energy fluence $F(E>1 \mathrm{GeV})$ is around a few $10^{-5} \mathrm{erg} \cdot \mathrm{cm}^{-2}$

\section{Conclusions}

The new experiment INCA II could observe GRBs in the range $E>1 G e V$ using the "single 

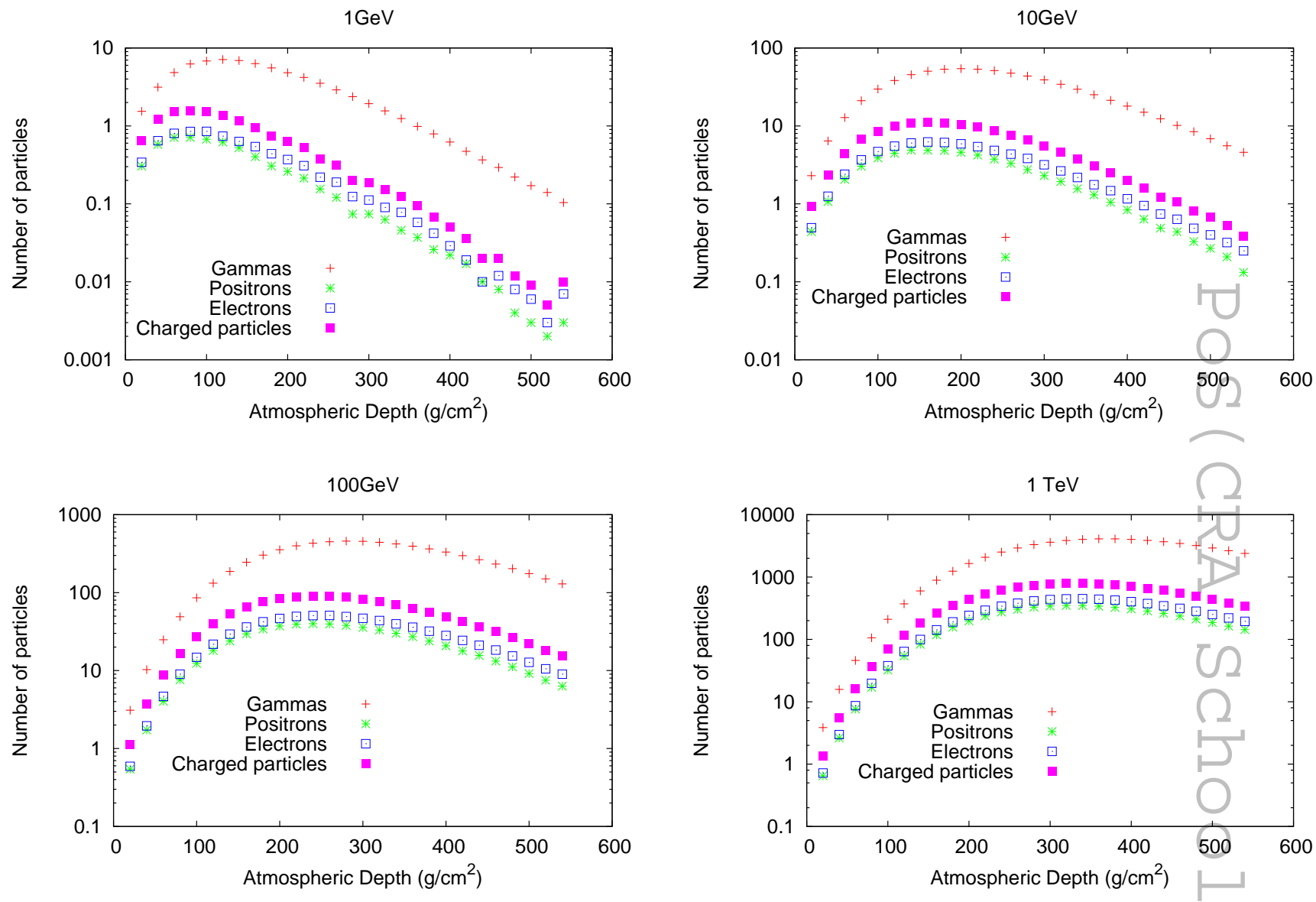

Figure 3: The longitudinal development of secondary particles from the primary gamma ray shower for $1 \mathrm{GeV}, 10 \mathrm{GeV}, 100 \mathrm{GeV}$ and $1 \mathrm{TeV}$ (vertical incidence).
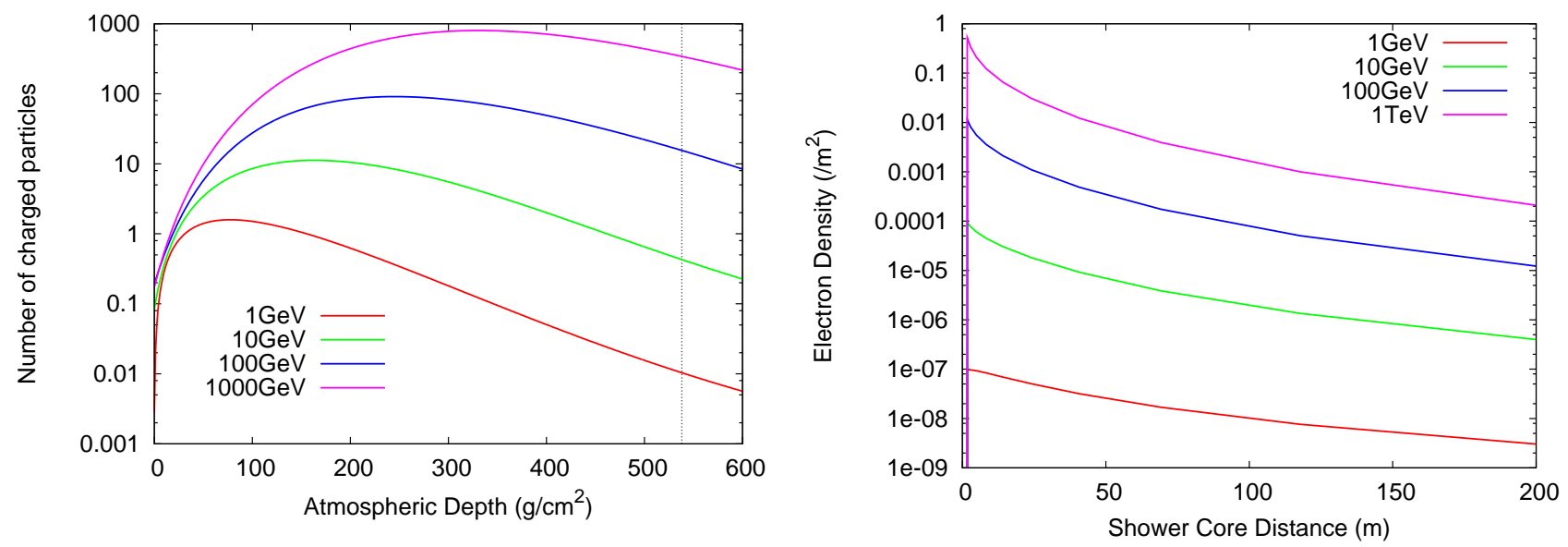

Figure 4: Right: Summary of longitudinal development of charged particles using the Gaisser-Hillas function. Left: The NKG lateral distribution. 


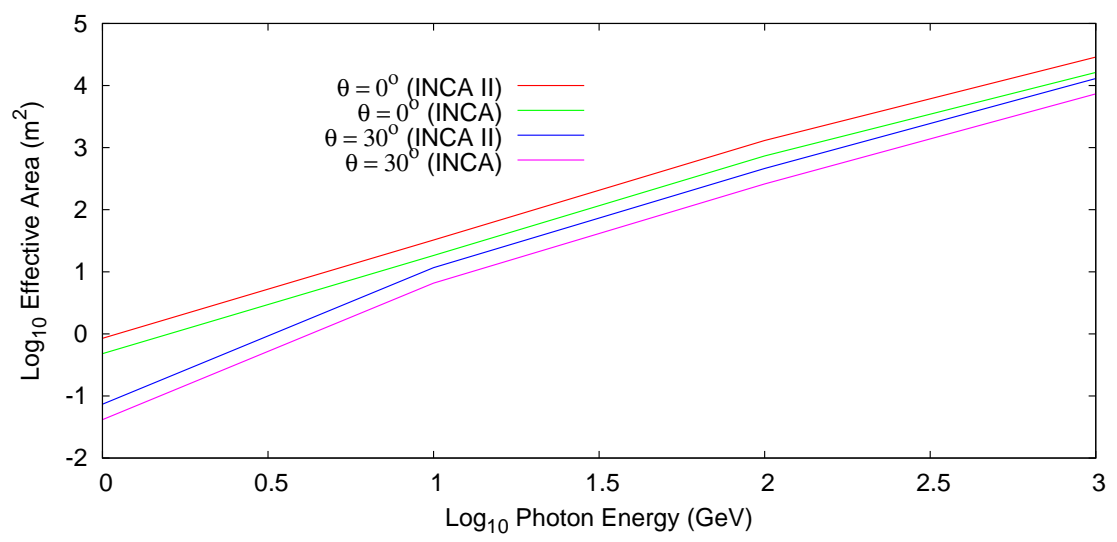

Figure 5: The effective area of INCA II compared to INCA, as a function of the photon energy for two zenith angles $\theta$.

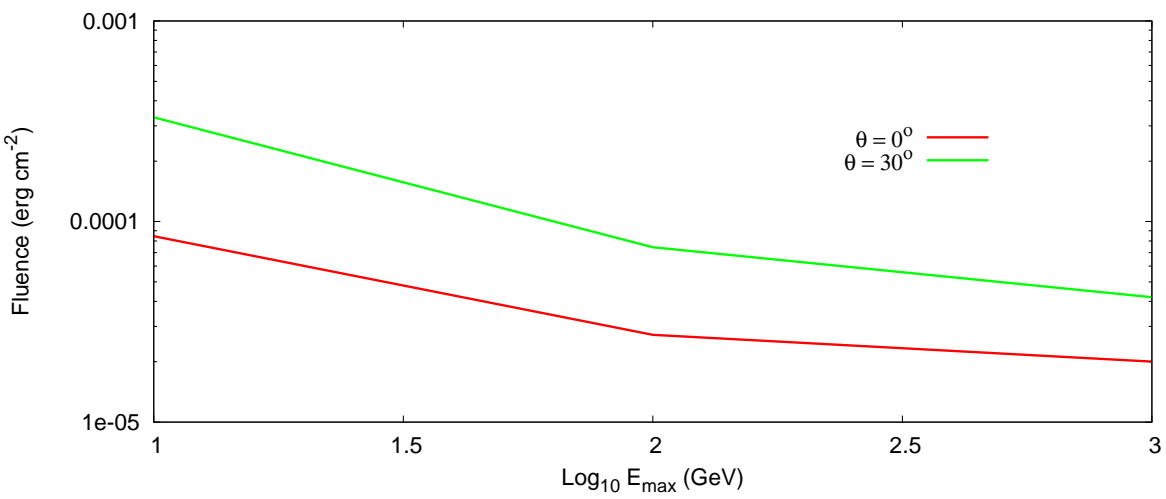

Figure 6: Minimum energy fluence that INCA II can observe in the range $1 \mathrm{GeV}<E<E_{\max }$, as a function of the maximun energy of the spectrum $E_{\max }$ for a detectable burst of time duration $\Delta t=1 \mathrm{~s}$ and for two zenith angles $\theta$.

particle" technique with minimum energy fluence as low as $F \sim 10^{-5} \mathrm{erg} \mathrm{cm}^{-2}$, if the spectral slope is $\alpha \sim 2$, comparable to that measured by satellite instruments. In spite of that the past experiment INCA reported upper limits on the energy fluence in the interval $10^{-5} \mathrm{erg} \mathrm{cm}^{2}$ to $10^{-2} \mathrm{erg} \mathrm{cm}^{2}$ in the energy range $1 \mathrm{GeV} \div 1 \mathrm{TeV}[5]$, we expect to propose new upper limits based in analysis data in order to compare with satellite observations and other experiments.

\section{Acknowledgements}

I am grateful to Prof. O. Saavedra and Y. Tsunesada for helpful comments and encouragements also to ICTP the financial support for my attendance to 4th School on Cosmic Rays and Astrophysics.

\section{References}

[1] CORSIKA web site: http://www-ik.fzk.de/corsika/

[2] Castellina A., et al., 1997, Il Nuovo Cimento, 20C, No. 2, 137. 
[3] Vernetto S. 2000 Astrop. Phys. 13.

[4] Aglietta M., et al.1996, ApJ, 469.

[5] Cabrera R., et al 1999, Astron. Astrophys. Suppl. 138. 